\title{
Pain score, desire for pain treatment and effect on pain satisfaction in the emergency department: a prospective, observational study
}

Judith E. van Zanden ${ }^{1 *}$ D, Susanne Wagenaar ${ }^{1}$, Jozine M. ter Maaten², Jan C. ter Maaten ${ }^{1}$ and Jack J. M. Ligtenberg ${ }^{1}$

\begin{abstract}
Background: Pain management in the Emergency Department has often been described as inadequate, despite proven benefits of pain treatment protocols. The aim of this study was to investigate the effectiveness of our current pain protocol on pain score and patient satisfaction whilst taking the patients' wishes for analgesia into account.

Methods: We conducted a 10-day prospective observational study in the Emergency Department. Demographics, pain characteristics, Numeric Rating Scale pain scores and the desire for analgesics were noted upon arrival at the Emergency Department. A second Numeric Rating Scale pain score and the level of patient satisfaction were noted 75-90 min after receiving analgesics. Student T-tests, Mann-Whitney $U$ tests and Kruskall-Wallis tests were used to compare outcomes between patients desiring vs. not desiring analgesics or patients receiving vs. not receiving analgesics. Univariate and multivariate logistic regression models were used to investigate associations between potential predictors and outcomes.

Results: In this study 334 patients in pain were enrolled, of which $43.7 \%$ desired analgesics. Initial pain score was the only significant predictive factor for desiring analgesia, and differed between patients desiring (7.01) and not desiring analgesics (5.14). Patients receiving analgesics (52.1\%) had a greater decrease in pain score than patients who did not receive analgesics (2.41 vs. 0.94). Within the group that did not receive analgesics there was no difference in satisfaction score between patients desiring and not desiring analgesics (7.48 vs. 7.54). Patients receiving analgesics expressed a higher satisfaction score than patients not receiving analgesics (8.10 vs. 7.53).

Conclusions: This study pointed out that more than half of the patients in pain entering the Emergency Department did not desire analgesics. In patients receiving analgesics, our pain protocol has shown to adequately treat pain, leading to a higher satisfaction for emergency health-care at discharge. This study emphasizes the importance of questioning pain score and desire for analgesics to prevent incorrect conclusions of inadequate pain management, as described in previous studies.
\end{abstract}

Keywords: Pain management, Emergency department, Oligoanalgesia, Pain satisfaction, Numeric rating scale

\footnotetext{
* Correspondence: j.e.van.zanden@umcg.nl

${ }^{1}$ Department of Emergency Medicine, University of Groningen, University

Medical Center Groningen, Groningen, The Netherlands

Full list of author information is available at the end of the article
}

(c) The Author(s). 2018 Open Access This article is distributed under the terms of the Creative Commons Attribution 4.0 International License (http://creativecommons.org/licenses/by/4.0/), which permits unrestricted use, distribution, and reproduction in any medium, provided you give appropriate credit to the original author(s) and the source, provide a link to the Creative Commons license, and indicate if changes were made. The Creative Commons Public Domain Dedication waiver (http://creativecommons.org/publicdomain/zero/1.0/) applies to the data made available in this article, unless otherwise stated. 


\section{Background}

As pain is a common complaint of patients visiting the Emergency Department (ED) [1], its early recognition and appropriate treatment is important. Experience of pain is one of the criteria on which quality of care in the ED is reviewed and a key factor in preventing unintentional, avoidable harm.

Since Marks and Sachar published a landmark paper about undertreatment of pain in the ED [2], this became a frequently investigated subject. The benefits of pain treatment protocols have been proven [3], yet pain management in the ED has often been described as inadequate $[1,4,5]$. This raises the question whether undertreatment of pain is caused by inadequate adherence to pain protocols, or, that 'oligoanalgesia' - a term to describe the phenomenon of undertreatment of pain - is overstated, as Green postulated in the Annals of Emergency Medicine [6]. Another question is whether pain should always be treated, given the fact that not all patients in pain desire analgesics $[7,8]$. To our knowledge, a prospective study on the effects of protocolized pain treatment on pain score and patient satisfaction, while taking desire for analgesics into account, has not been previously published.

The main goal of our study was to prospectively investigate the effectiveness of our current pain protocol on pain score and patient satisfaction in our ED; the desire for analgesia by the patient is hereby taken into account. 'Effectiveness' of pain treatment was defined as a decrease in pain score and increased level of satisfaction with care. A pain score reduction of $>2$ Numeric Rating
Scale (NRS) was considered clinically relevant $[9,10]$. Secondary goals were to evaluate arguments for refusing analgesics and reasons for not receiving analgesics, despite patients' expressed desire for pain relief. We hypothesized that patients receiving analgesics would have a stronger decrease in pain score and a higher score of patient satisfaction compared to patients who did not receive analgesics.

\section{Methods}

This study had a prospective, observational design and was conducted at the ED of the University Medical Center Groningen (UMCG). The ED of this tertiary teaching hospital has an annual volume of approximately 32.000 patients, of which around 22.000 are estimated to be non-trauma patients. At the onset of the study, an analgesic protocol was present in the ED (Fig. 1). Data was obtained by study personnel filling out a comprehensive questionnaire for patients $>18$ years old visiting the ED. This study was approved by the institutional review board of the UMCG. All patients signed the informed consent to participate in this study.

All patients with complaints of recent pain $(<3$ months) were questioned. Exclusion criteria were patients $<18$ years, no recent pain $(<3$ months), patients in a life-threatening situation, patients with impaired mental status or neuropsychiatric diseases leading to inability to fill in the questionnaire (e.g. patients suffering from delirium, dementia or psychosis), a language barrier or absence of informed consent.

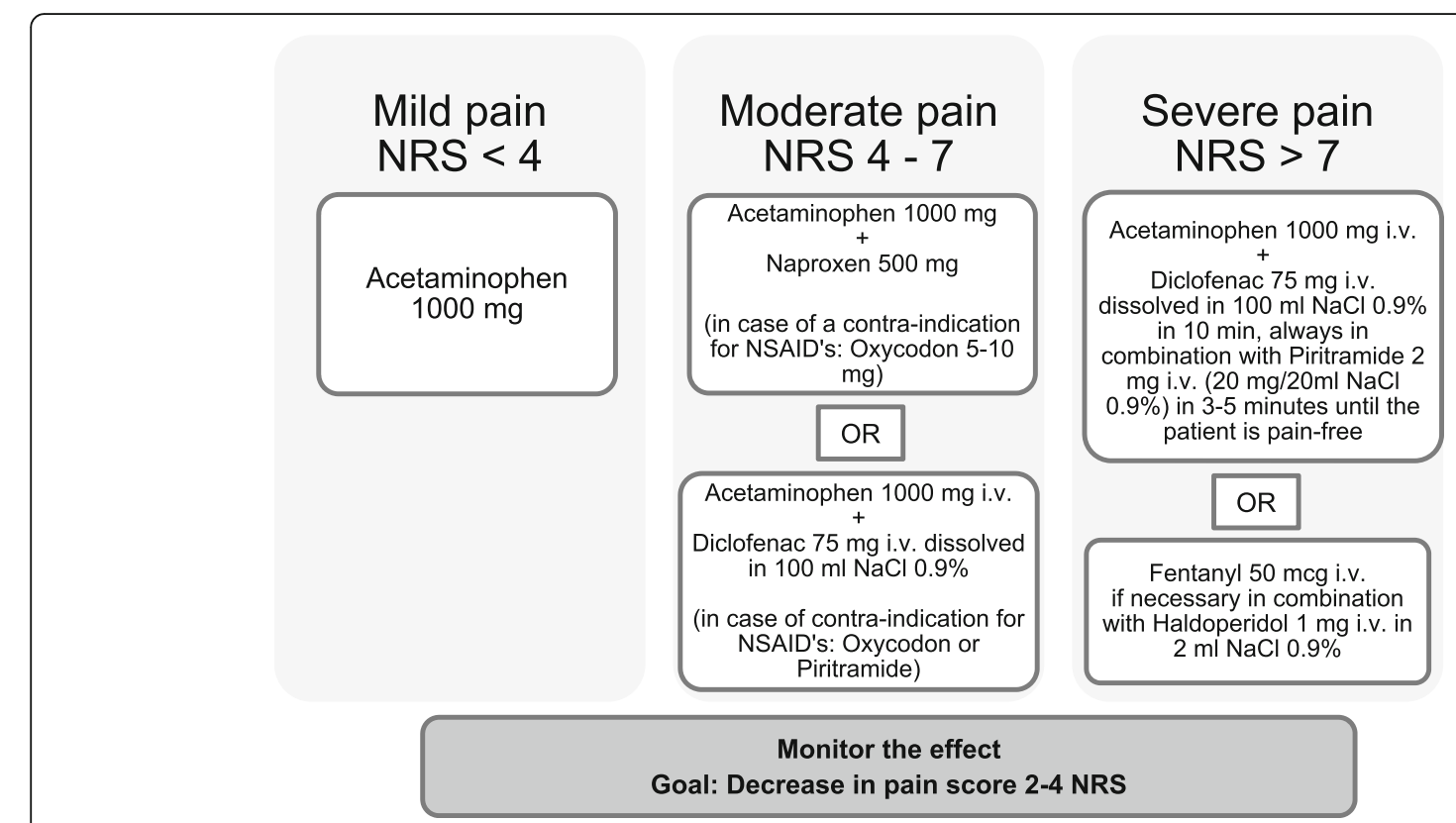

Fig. 1 The University Medical Center Groningen pain protocol 
We used a manual questionnaire for a continuous period of 10 days, $24 \mathrm{~h}$ a day, to collect data for every patient visiting the ED.

During their stay, all patients were questioned twice. Immediately after triage of the patient, data was collected on demographics (age, sex), triage colour code (Table 1), allergy for analgesics, medical specialty of referral, pain characteristics (location, duration, type and character of pain) and pain score at that time, using the Numeric Rating Scale, ranging from 0 (no pain) to 10 (worst pain the patient can imagine). We checked whether the patient had already received analgesics before arriving at the hospital, e.g. self-medication or analgesics given by the general physician or ambulance staff. Furthermore, we asked whether the patient desired pain management. In case there was no desire for analgesics, the patient was asked about the reason. The second part of the questionnaire was filled in 75-90 min after taking the initial questionnaire. First, we checked if the patient had received analgesics in the ED and the type and dose of analgesics given. An analgesic was defined as any drug prescribed with the aim to reduce pain, such as acetaminophen, nonsteroidal anti-inflammatory drugs (NSAID) and opioids, as described in the analgesic ladder of the World Health Organization (WHO) [11]. Besides that, in accordance with previous articles published on this subject, drugs having a possible indirect effect on pain level were also included. Those were nitroglycerin for chest pain, proton pump inhibitors (PPI) for abdominal pain and benzodiazepines $[7,8]$. If the patient did not receive any analgesics but had previously indicated a desire to receive analgesics, we asked the patient and the nurse why analgesics were not administered. The patient was asked again to rate pain severity on the Numeric Rating Scale. Lastly, the patient was asked to express their satisfaction on how their entire pain management was executed, rated on a Numeric Rating Scale, ranging from 0 (very dissatisfied) to 10 (very satisfied).

Primary outcomes were change in pain score and patient satisfaction score. Secondary outcomes were reasons to refuse analgesics and identifying why patients did not receive analgesics, despite desire for pain relief.

Table 1 Triage colour code, as used in the University Medical Center Groningen

\begin{tabular}{llll}
\hline Urgency & Name & Colour & $\begin{array}{l}\text { Maximum waiting } \\
\text { time }\end{array}$ \\
\hline 1 & Immediately & Red & 0 min \\
2 & Highly urgent & Orange & $10 \mathrm{~min}$ \\
3 & Urgent & Yellow & $60 \mathrm{~min}$ \\
4 & Standard & Green & $120 \mathrm{~min}$ \\
5 & Not urgent & Blue & $240 \mathrm{~min}$ \\
\hline
\end{tabular}

After completion of the study, all blinded questionnaires were manually entered in SPSS and checked by two investigators.

\section{Statistical analysis}

Binominal and categorical data are presented as frequencies of occurrence and percentages. Continuous variables are presented as means and standard deviations (SD). The student T-test or Mann-Whitney U test was used to compare means between patients desiring vs. not desiring analgesics or patients receiving vs. not receiving analgesics. To compare between multiple groups, the Kruskall-Wallis test was used, followed by post hoc Mann-Whitney U tests. $P$-values were described to demonstrate differences between groups, with $p<0.05$ considered significant.

To investigate associations between potential predictors and outcomes, logistic regressions were conducted. Factors that showed to be significant in univariate logistic regressions were included in a multivariate logistic regression model. Associations are presented by odds ratios (OR) and confidence intervals (CI). P-values of < 0.05 were again considered significant. All analyses were performed using IBM SPSS Statistics version 22.

\section{Results}

The total number of patients that visited the ED during the 10 days of inclusion was 797 (Fig. 2). Of those, 199 patients had no recent pain. Furthermore, 93 patients were excluded for being $<18$ years old, 12 patients were excluded for being in a life-threatening situation, 28 patients had an altered mental status or neuropsychiatric disease, in 13 patients a language barrier was present, 14 patients did not give informed consent and 1 patient could not be questioned due to a too high infection risk. In addition, 14 patients were excluded because they participated earlier in the study, 74 patients were discharged before completing the questionnaire and 15 patients were not questioned because of organizational issues. In total, 463 patients were excluded from the study.

\section{Characteristics of study subjects}

The total number of patients included during the 10-day study was 334 . The mean age was 49 years and $53 \%$ were male. The overall mean initial pain score was 5.96 NRS (SD 2.28). Half of the patients had already taken analgesics before entering the ED (50.9\%) and $5.1 \%$ of the patients indicated to have an allergy for an analgesic. Patient and pain characteristics are outlined in Table 2.

\section{Effect of pain protocol on pain score}

Of the 334 enrolled patients, 146 (43.7\%) patients desired analgesics upon arrival at the ED. Initial pain scores were higher in patients with a desire for 


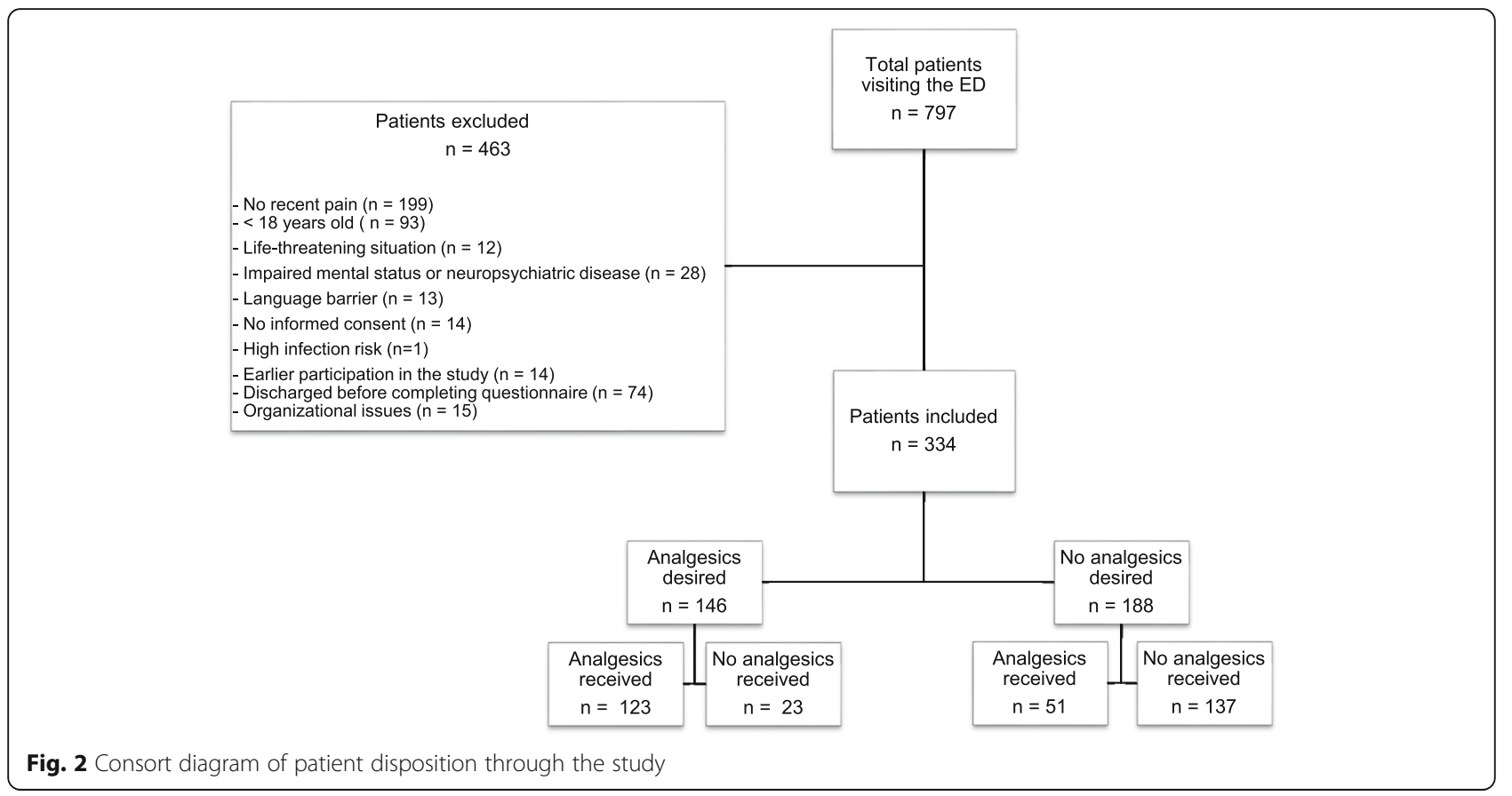

analgesics compared to patients who did not wish analgesics (NRS score 7.01 [95\% CI 6.70-7.33] vs. NRS score 5.14 [95\% CI 4.82-5.45], Table 2). Desire for analgesics did not depend on factors such as gender, age, specialty of referral, pain character, pre-ED analgesics or known analgesic allergy. The variables triage colour code, pain location, pain duration and pain pattern (constant or intermittent pain) showed significant differences between the two groups (Table 2). Patients assigned to a group with a more urgent colour code were more likely to desire analgesics and patients with chest pain were less likely to desire analgesics. The duration of pain showed a trend towards medium pain duration (6-12 h) and a desire for analgesics. Patients suffering from constant pain were more likely to desire analgesics compared to patients experiencing intermittent pain (OR 1.89 [95\% CI 1.10-3.26]).

The factors triage colour code, pain location, pain pattern and mean initial pain score were univariately significantly associated with the desire for analgesics and were the eligible factors for the multivariate model. In multivariate analysis, only the initial pain score was a significant predictive factor for desiring analgesia (Table 3).

Of all the patients in pain, 174 patients $(52.1 \%)$ received analgesics. The most commonly prescribed analgesics were acetaminophen (50.2\%), NSAID's (13.9\%) and opioids (21.2\%) (Table 4).

The number of patients receiving analgesics was greater than the number of patients who initially desired analgesics (174 vs. 146 patients). Of the 188 patients that initially refused analgesics, 51 patients had required and received analgesics at the time of the second questionnaire. Of the patients initially desiring analgesics, 23 patients did not receive them (Fig. 2). In order to accurately present the results, those two groups were temporarily excluded. In total, 260 patients (123 patients that desired and received analgesics and 137 patients that refused and did not receive analgesics) were included in the following analyses. Patients who received analgesics during their stay in the ED had significantly higher initial pain scores than those who did not receive analgesics (NRS score 7.09 [95\% CI 6.76-7.42] vs. NRS score 4.82 [95\% CI 4.46-5.19]). Pain scores measured 75-90 min after arrival decreased significantly in patients who received analgesics, with a reduction of 2.41 NRS (95\% CI 2.02-2.79). The pain score of patients who received analgesics decreased more than patients who did not receive analgesics; the group of patients not receiving analgesics showed a reduction in pain score of 0.94 NRS ([95\% CI 0.62-1.25], Table 5).

\section{Effect of the pain protocol on patient satisfaction}

Overall, all patients included in this study expressed a relatively high satisfaction of 7.83 (95\% CI 7.66-7.99) for pain management. The majority of patients (67.1\%) was satisfied (patient satisfaction score between 6 and 8 NRS), and $25.7 \%$ of the patients were even highly satisfied (patient satisfaction score 9-10). Only $7.2 \%$ of all patients included were dissatisfied (patient satisfaction score $<6$ NRS). 
Table 2 Patient and pain characteristics with subdivision in desire for analgesics

\begin{tabular}{|c|c|c|c|}
\hline \multirow[t]{2}{*}{ Patient factors } & Desire for analgesics & No desire for analgesics & \multirow[t]{2}{*}{ p-value } \\
\hline & $(n=146)$ & $(n=188)$ & \\
\hline \multicolumn{4}{|l|}{ Gender } \\
\hline Male $(n=177)$ & $72(40.7 \%)$ & $105(59.3 \%)$ & \multirow[t]{2}{*}{0.235} \\
\hline Female $(n=157)$ & $74(47.1 \%)$ & $83(52.9 \%)$ & \\
\hline Mean age (SD) & $48.38(18.44)$ & $49.06(18.39)$ & 0.675 \\
\hline \multicolumn{4}{|l|}{ Triage colour code } \\
\hline Blue $(n=7)$ & $2(28.6 \%)$ & $5(71.4 \%)$ & \multirow[t]{5}{*}{0.010} \\
\hline Green $(n=130)$ & $45(34.6 \%)$ & $85(65.4 \%)$ & \\
\hline Yellow $(n=175)$ & $84(48 \%)$ & 91 (52\%) & \\
\hline Orange $(n=18)$ & $13(72.2 \%)$ & $5(27.8 \%)$ & \\
\hline $\operatorname{Red}(n=4)$ & $2(50 \%)$ & $2(50 \%)$ & \\
\hline \multicolumn{4}{|l|}{ Specialty of referral } \\
\hline Traumatology $(n=78)$ & $38(48.7 \%)$ & $40(51.3 \%)$ & \multirow[t]{9}{*}{0.121} \\
\hline Internal medicine $(n=50)$ & $22(44 \%)$ & $28(56 \%)$ & \\
\hline Emergency medicine $(n=26)$ & $12(46.2 \%)$ & $14(53.8 \%)$ & \\
\hline Cardiology $(n=45)$ & $12(26.7 \%)$ & $33(73.3 \%)$ & \\
\hline Abdominal surgery $(n=32)$ & $17(53.1 \%)$ & $15(46.9 \%)$ & \\
\hline Orthopedics $(n=32)$ & $9(28.1 \%)$ & $23(71.9 \%)$ & \\
\hline Lung diseases $(n=16)$ & $7(43.8 \%)$ & $9(56.3 \%)$ & \\
\hline Neurology $(n=15)$ & $9(60 \%)$ & $6(40 \%)$ & \\
\hline Other $(n=40)$ & $20(50 \%)$ & $20(50 \%)$ & \\
\hline \multicolumn{4}{|l|}{ Pain location } \\
\hline Upper extremities $(n=47)$ & $18(38.3 \%)$ & $29(61.7 \%)$ & \multirow[t]{9}{*}{0.033} \\
\hline Lower extremities ( $n=54)$ & $23(42.6 \%)$ & $31(57.4 \%)$ & \\
\hline Abdomen $(n=75)$ & $42(56 \%)$ & $33(44 \%)$ & \\
\hline Head/face $(n=24)$ & $13(54.2 \%)$ & $11(45.8 \%)$ & \\
\hline $\operatorname{Back}(n=12)$ & $7(58.3 \%)$ & $5(41.7 \%)$ & \\
\hline Chest $(n=54)$ & $14(25.9 \%)$ & $40(74.1 \%)$ & \\
\hline Neck $(n=3)$ & $1(33.3 \%)$ & $2(66.7 \%)$ & \\
\hline More than 1 location $(n=47)$ & $23(48.9 \%)$ & $24(51.1 \%)$ & \\
\hline Other $(n=18)$ & $5(27.8 \%)$ & $13(72.2 \%)$ & \\
\hline \multicolumn{4}{|l|}{ Pain duration } \\
\hline$<2 \mathrm{~h}(n=68)$ & $32(47.1 \%)$ & $36(52.9 \%)$ & \multirow[t]{5}{*}{0.047} \\
\hline $2-6 \mathrm{~h}(n=52)$ & 27 (51.9\%) & $25(48.1 \%)$ & \\
\hline $6-12 \mathrm{~h}(n=31)$ & $19(61.3 \%)$ & $12(38.7 \%)$ & \\
\hline $12 \mathrm{~h}-3$ days $(\mathrm{n}=78)$ & $32(41.0 \%)$ & $46(59.0 \%)$ & \\
\hline$>3$ days $(n=105)$ & $36(34.3 \%)$ & $69(65.7 \%)$ & \\
\hline \multicolumn{4}{|l|}{ Pain pattern } \\
\hline Constant $(n=259)$ & $122(47.1 \%)$ & $137(52.9 \%)$ & \multirow[t]{2}{*}{0.020} \\
\hline Intermittent $(n=75)$ & $24(32 \%)$ & $51(68 \%)$ & \\
\hline \multicolumn{4}{|l|}{ Pain character } \\
\hline Sharp $(n=121)$ & $58(47.9 \%)$ & $63(52.1 \%)$ & \multirow[t]{3}{*}{0.425} \\
\hline Pressing $(n=66)$ & $27(40.9 \%)$ & $39(59.1 \%)$ & \\
\hline Nagging $(n=62)$ & $31(50 \%)$ & $31(50 \%)$ & \\
\hline
\end{tabular}


Table 2 Patient and pain characteristics with subdivision in desire for analgesics (Continued)

\begin{tabular}{lll}
\hline Patient factors & $\begin{array}{l}\text { Desire for analgesics } \\
(n=146)\end{array}$ & $\begin{array}{l}\text { No desire for analgesics } \\
(n=188)\end{array}$ \\
\hline Burning $(n=18)$ & $5(27.8 \%)$ & $13(72.2 \%)$ \\
Cramping $(n=7)$ & $3(42.9 \%)$ & $4(57.1 \%)$ \\
More than 1 answer $(n=48)$ & $19(39.6 \%)$ & $29(60.4 \%)$ \\
Other $(n=12)$ & $3(25 \%)$ & $9(75 \%)$ \\
Mean initial pain score (SD) & $7.01(1.94)$ & $5.14(2.19)$ \\
Pre-ED analgesics & & $87(51.2 \%)$ \\
Yes $(n=170)$ & $83(48.8 \%)$ & $101(61.6 \%)$ \\
No $(n=164)$ & $63(38.4 \%)$ & \\
Analgesia allergy & & $9(52.9 \%)$ \\
Yes $(n=17)$ & $8(47.1 \%)$ & $179(56.5 \%)$ \\
No $(n=317)$ & $138(43.5 \%)$ & 0.029 \\
\hline
\end{tabular}

P-values show differences of the whole factor group in relation to desire vs. no desire for analgesia

After only analyzing patients who received analgesics in compliance with their initial wish, results show that patients receiving analgesics were significantly more satisfied than patients who did not receive analgesics (satisfaction score 8.06 [95\% CI 7.80-8.31 vs. 7.54 [95\% CI 7.24-7.84]).

\section{Reasons for refusing analgesics}

The most common reason for not wanting analgesics, even though in pain, was bearable pain $(57.4 \%)$, wanting to know the diagnosis first (10\%) and using analgesics before visiting the ED (8\%).

\section{Reasons for not receiving analgesics and the effect on patient satisfaction levels}

As stated before, not all patients received analgesia according to their initial wish. Of the 146 patients desiring pain management, 23 patients did not receive analgesics. After performing subgroup analyses on patient satisfaction scores, results show that within the group that did not receive analgesics, patients who initially desired analgesics were not more dissatisfied than patients that did not desire analgesics (mean patient satisfaction score 7.48 [95\% CI 6.90-8.06] vs. 7.54 [96\% CI 7.24-7.84], Fig. 3). This result is important in investigating whether analgesics were incorrectly withheld from patients. In this cohort, the main reasons for not receiving analgesics included receiving an alternative non-pharmacological pain treatment such as splinting or applying ice or heat (31\%), crowding in the ED (13\%) and the clinical judgement of the nurse (13\%). After correction for non-pharmacological pain treatment, $6.6 \%$ of the 334 patients in pain did not receive analgesics or a non-pharmacological pain treatment, despite their expressed desire.

\section{Discussion}

Pain management is an important criterion of quality of care given in the ED and its adequacy has therefore been often discussed in literature.

According to our initial hypothesis, our study shows that the pain score in patients who received analgesics decreased more than in patients who did not receive analgesics. Patients who received analgesics were also significantly more satisfied than patients who did not receive analgesics. Those differences are in our study unlikely to be attributed to 'undertreatment of pain' in patients that did not receive analgesics. An important question in investigating 'undertreatment of pain' or 'oligoanalgesia' is whether all patients in pain desire analgesia. Previous research shows that nearly half of the patients in pain decline analgesia, which is in agreement with our results $[7,8]$ : of all the patients entering the ED in pain, less than half desired analgesics in our study. In those patients, 'bearable pain' was the most common reason for refusing analgesics. More urgent cases, medium pain duration of 6-12 h and constant pain were factors more associated with desiring analgesics, describing possible reasons why and when analgesics are desired.

Of patients that did not receive analgesics, the majority $(86 \%)$ did not desire analgesics. Also, there was no difference in satisfaction between those patients and patients that did not receive analgesics despite their wish, suggesting that analgesics were not incorrectly withheld from patients. According to Allione et al., almost 10\% of the patients do not receive analgesics despite their expressed desire [8], however, the reason for this was 
Table 3 Factors associated with a desire for analgesics (univariate and multivariate analysis)

\begin{tabular}{|c|c|c|c|c|}
\hline & Univariate analysis & & Multivariate analysis & \\
\hline Variable & OR $(95 \% \mathrm{Cl})$ & $p$-value & OR $(95 \% \mathrm{Cl})$ & p-value \\
\hline Gender & & 0.236 & & \\
\hline Male & Reference & & & \\
\hline Female & $1.30(0,84-2.01)$ & & & \\
\hline Age & $0.10(0.99-1.01)$ & 0.737 & & \\
\hline Triage colour code & & 0.021 & & 0.139 \\
\hline Blue & $0.40(0.03-5.15)$ & & $1.23(0.07-0.76)$ & \\
\hline Green & $0.53(0.72-3.89)$ & & $1.65(0.19-4.61)$ & \\
\hline Yellow & $0.92(0.13-6.70)$ & & $2.98(0.34-5.83)$ & \\
\hline Orange & $2.60(0.28-3.81)$ & & $6.05(0.54-8.08)$ & \\
\hline Red & Reference & & Reference & \\
\hline Specialty of referral & & 0.14 & & \\
\hline Traumatology & Reference & & & \\
\hline Internal medicine & $0,83(0.41-1.69)$ & & & \\
\hline Emergency medicine & $0.90(0.37-2.20)$ & & & \\
\hline Cardiology & $0.38(0.17-0.85)$ & & & \\
\hline Abdominal surgery & $1.19(0.52-2.72)$ & & & \\
\hline Orthopedics & $0.41(0.17-1.00)$ & & & \\
\hline Lung diseases & $0.82(0.28-2.42)$ & & & \\
\hline Neurology & $1.58(0.51-4.86)$ & & & \\
\hline Other & $1.05(0.49-2.26)$ & & & \\
\hline Pain location & & 0.040 & & 0.078 \\
\hline Upper extremities & Reference & & Reference & \\
\hline Lower extremities & $1.20(0.54-2.66)$ & & $1.29(0.53-3.09)$ & \\
\hline Abdomen & $2.05(0.97-4.32)$ & & $1.68(0.74-3.79)$ & \\
\hline Head/face & $1.90(0.70-5.15)$ & & $2.94(0.97-8.93)$ & \\
\hline Back & $2.26(0.62-8.19)$ & & $1.99(0.48-8.21)$ & \\
\hline Chest & $0.56(0.24-1.31)$ & & $0.68(0.27-1.70)$ & \\
\hline Neck & $0.81(0.07-9.54)$ & & $0.45(0.03-6.67)$ & \\
\hline More than 1 location & $1.54(0.68-3.51)$ & & $2.29(0.92-5.71)$ & \\
\hline Other & $0.62(0.19-2.03)$ & & $0.62(0.17-2.21)$ & \\
\hline Pain duration & & 0.051 & & \\
\hline$<2 \mathrm{~h}$ & $1.70(0.91-3.18)$ & & & \\
\hline $2-6 h$ & $2.07(1.05-4.07)$ & & & \\
\hline $6-12 \mathrm{~h}$ & $3.04(1.33-6.94)$ & & & \\
\hline $12 \mathrm{~h}-3$ days & $1.33(0.73-2.44)$ & & & \\
\hline$>3$ days & Reference & & & \\
\hline Pain pattern & & 0.021 & & 0.314 \\
\hline Constant & $1.89(1.10-3.26)$ & & $1.38(0.74-2.58)$ & \\
\hline Intermittent & Reference & & Reference & \\
\hline Pain character & & 0.447 & & \\
\hline Sharp & Reference & & & \\
\hline Pressing & $0.75(0.41-1.38)$ & & & \\
\hline Nagging & $1.09(0.59-2.00)$ & & & \\
\hline
\end{tabular}


Table 3 Factors associated with a desire for analgesics (univariate and multivariate analysis) (Continued)

\begin{tabular}{|c|c|c|c|c|}
\hline & Univariate analysis & & Multivariate analysis & \\
\hline Burning & $0.42(0.14-1.24)$ & & & \\
\hline Cramping & $0.82(0.18-3.80)$ & & & \\
\hline More than 1 answer & $0.71(0.36-1.40)$ & & & \\
\hline Other & $0.36(0.08-1.40)$ & & & \\
\hline Mean initial pain score & $1.54(1.36-1.74)$ & 0.000 & $1.56(1.37-1.77)$ & 0.000 \\
\hline Pre-ED analgesics & & 0.056 & & \\
\hline No & Reference & & & \\
\hline Yes & $1.53(0.99-2.36)$ & & & \\
\hline Analgesia allergy & & 0.775 & & \\
\hline No & Reference & & & \\
\hline Yes & $1.15(0.43-3.07)$ & & & \\
\hline
\end{tabular}

not described. Our study showed that receiving a non-pharmacological pain treatment was the most common reason for not receiving analgesics. After correcting for non-pharmacological pain treatment, only $6.6 \%$ of the patients did not receive analgesia or a non-pharmacological pain treatment despite their expressed desire for pain relief. Not correcting for the patients' wish for analgesia might lead to low rates of analgesic administration and thus to incorrect conclusions with regard to inadequate pain management, reporting undertreatment percentages ranging from 20 to $50 \%$ as described before in literature [12].

Nevertheless, it has to be mentioned that despite the statistically significant differences, one might wonder if a difference of 0.52 in patient satisfaction score is clinically relevant. Previous studies have investigated the association between pain scores and patient satisfaction and stated that pain relief on itself is not the only factor affecting patient satisfaction [13, 14]. McNeill et al. showed a negative correlation between pain scores and patient satisfaction [15], while Svensson et al. showed a

Table 4 Overview of prescribed types of analgesics

\begin{tabular}{ll}
\hline Administered drug & No. of patients $(n=174)$ \\
\hline Acetaminophen & $64(36.8 \%)$ \\
NSAID & $4(2.3 \%)$ \\
Opioid & $19(10.9 \%)$ \\
Acetaminophen + NSAID & $23(13.2 \%)$ \\
Acetaminophen + NSAID + opioid & $4(2.3 \%)$ \\
Acetaminophen + opioid & $25(14.4 \%)$ \\
NSAID + opoid & $1(0.6 \%)$ \\
Nitroglycerin & $9(5.1 \%)$ \\
Benzodiazepine & $1(0.6 \%)$ \\
Others & $24(13.8 \%)$ \\
\hline
\end{tabular}

high patient satisfaction score despite high pain scores [16]. Phillips et al. concluded from their study that patient satisfaction should be addressed as an independent variable in assessing pain management [12].

Another important limitation of many earlier studies on 'oligoanalgesia' is their retrospective nature. Since pain perception is a subjective phenomenon, the presence or severity of pain should be determined by asking the patient at the moment of pain itself. Estimating the pain score, without asking the patient or asking the patient in retrospective, will not reliably reveal the real severity of pain [17]. Also, the number of patients receiving analgesics has more often been investigated, but rarely the actual effects of analgesics on lowering the pain score. A review of literature on oligoanalgesia stated that inconsistency and inadequacy of pain management is caused by multiple reasons including a lack of education in pain management, inadequate management programs to evaluate pain management, the clinicians' attitude towards opioids and disbelief of pain reporting [18]. A generally accepted pain protocol is therefore important, providing guidelines aiming to prevent an inconsistency in pain treatment. Our study showed an overall pain reduction of 2.17 NRS in the group of patients treated with analgesia according to our pain protocol. Since a pain reduction of $>2$ NRS is considered clinically relevant $[9,10]$, the current pain protocol can be considered adequate.

\section{Limitations of the study}

Measuring severity of pain among ED patients can be challenging since pain perception is considered a subjective phenomenon. A retrospective way of investigating pain management will not provide a reliable picture [17]. However, a prospective way also has its limitations. The questionnaires were filled in by researchers questioning the patient. Some patients may have been inclined to 
Table 5 Reduction in pain score in patients receiving analgesics vs. patients not receiving analgesics

\begin{tabular}{llll}
\hline & Analgesics received $(n=123)$ & No analgesics received $(n=137)$ & $p$-value \\
\hline Initial pain score $(95 \% \mathrm{Cl})$ & $7.09(6.76-7.42)$ & $4.82(4.46-5.19)$ & 0.00 \\
Pain score after $75-90 \mathrm{~min}(95 \% \mathrm{Cl})$ & $4.68(4.23-5.14)$ & $3.89(3.46-4.32)$ & 0.013 \\
$\triangle$ Pain score $(95 \% \mathrm{Cl})$ & $2.41(2.02-2.79)$ & $0.94(0.62-1.25)$ & 0.00 \\
\hline
\end{tabular}

provide responses based on an expectation of a correct response; thus skewing data. Secondly, the study was conducted under optimal circumstances of a working pain protocol. The presence of the researchers in the work field might have induced a 'Hawthorne effect', reminding nurses to pay more attention to pain management. Some patients expressed their desire for analgesics only to the researcher. In this case, the nurses were informed for ethical reasons. Further research will focus on the adherence to the pain management protocol under ordinary working circumstances and overcrowding.

An important challenge in investigating patient satisfaction on pain management was the subgroup of patients where no analgesics were given. For that reason, all patients were asked to rate the overall management routine regarding pain treatment in the Emergency Department, and not only the effect of the therapeutics itself. Nevertheless, it is possible that less attention was given to patients who initially did not desire analgesics, possibly causing the differences in satisfaction scores between the two groups. Patient satisfaction score was expressed as one overall grade in our questionnaire. Thus, specific reasons for expression of (dis)satisfaction regarding e.g. waiting time and communication, were not investigated.

This study is limited to a single, tertiary university hospital in Groningen, the north of the Netherlands. ED's of all Dutch hospitals adhere to the same guidelines for pain management [19]. Therefore, pain protocols used in other Dutch hospitals can be considered as comparable. However, e.g. patient demographics can differ between ED's. Our results should therefore not be generalized to other, dissimilar settings.

\section{Conclusions}

In summary, our results show that less than half of the patients in pain entering the ED desire analgesics. Patients receiving analgesics were adequately treated according to the pain protocol, showing a clinically relevant reduction in pain score and higher satisfaction scores at discharge compared to patients who did not receive analgesics. Patients who did not receive analgesics despite their expressed wish were not incorrectly withheld from pain treatment, since satisfaction scores were comparable to patients who did not desire and did not receive analgesics. Additionally, most of the patients who did not receive pain medication despite desiring

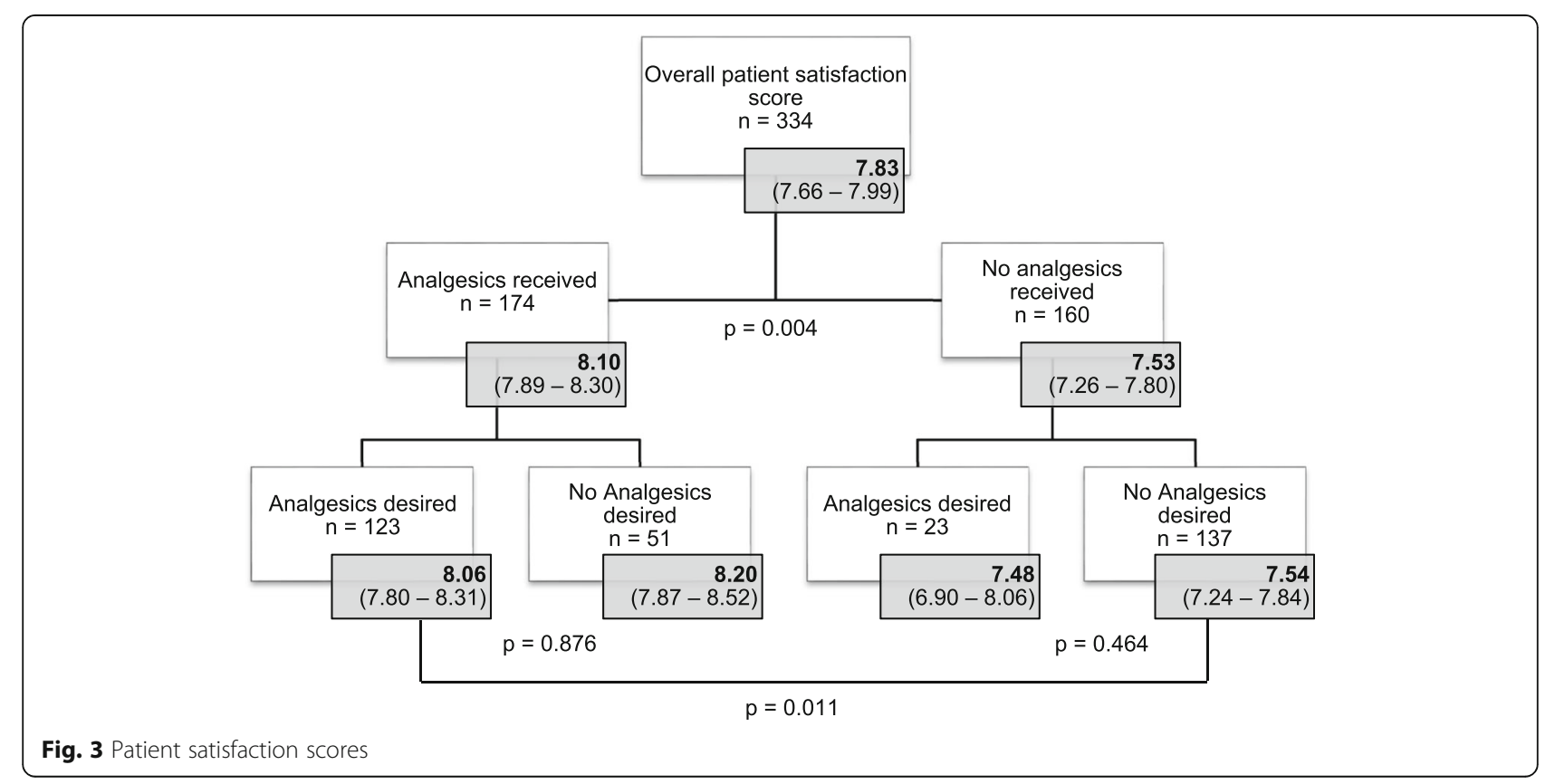


analgesics, received a non-pharmacological pain treatment. This study demonstrates that in this ED setting with a pain protocol in use, pain is adequately treated, and emphasizes the importance of taking the patients' wish for analgesia into account when investigating 'oligoanalgesia' in the ED.

\section{Abbreviations}

Cl: Confidence Interval; ED: Emergency Department; NRS: Numeric Rating Scale; NSAID: Nonsteroidal Anti-Inflammatory Drugs; OR: Odds Ratio; PPI: Proton pump inhibitor; SD: Standard Deviation; UMCG: University Medical Center Groningen; WHO: World Health Organization

\section{Acknowledgements}

The authors are very grateful to all doctors and nurses from the Emergency Department at the University Medical Center Groningen hospital, who contributed to this study.

\section{Funding}

Not applicable.

\section{Availability of data and materials}

The datasets used and/or analyzed during the current study are available through the corresponding author on reasonable request.

\section{Authors' contributions}

$J Z$ and JCM conceived the study and designed the trial. JL and JCM supervised the conduct of the trial and data collection, performed by JZ and SW. JJM provided statistical advice analyzing the data. JZ analyzed the data and drafted the article. All authors read and approved the final manuscript.

\section{Ethics approval and consent to participate}

All patients gave written informed consent for participation in the study which was approved by the institutional review board of the UMCG.

\section{Consent for publication}

Not applicable, since data was used anonymously.

\section{Competing interests}

The authors declare that they have no competing interests.

\section{Publisher's Note}

Springer Nature remains neutral with regard to jurisdictional claims in published maps and institutional affiliations.

\section{Author details}

${ }^{1}$ Department of Emergency Medicine, University of Groningen, University Medical Center Groningen, Groningen, The Netherlands. ${ }^{2}$ Department of Cardiology, University of Groningen, University Medical Center Groningen, Groningen, The Netherlands.

Received: 28 February 2018 Accepted: 23 October 2018

Published online: 08 November 2018

\section{References}

1. Tanabe P, Buschmann M. A prospective study of ED pain management practices and the patient's perspective. J Emergency Nursing. 1999;25(3): 171-7.

2. Marks RM, Sachar EJ. Undertreatment of medical inpatients with narcotic analgesics. Ann Intern Med. 1973;78(2):173-81.

3. Stephan F-P, Nickel CH, Martin JS, Grether D, Delport-Lehnen K, Bingisser R. Pain in the emergency department: adherence to an implemented treatment protocol. Swiss Med Wkly. 2010;140(23-24):341-7.

4. Wilson JE, Pendleton JM. Oligoanalgesia in the emergency department. Am J Emerg Med. 1989;7(6):620-3.

5. Selbst SM, Clark M. Analgesic use in the emergency department. Ann Emerg Med. 1990;19(9):1010-3.

6. Green SM. There is oligo-evidence for oligoanalgesia. Ann Emerg Med. 2012;60(2):212-4.
7. Singer AJ, Garra G, Chohan JK, Dalmedo C, Thode HC. Triage pain scores and the desire for and use of analgesics. Ann Emerg Med. 2008; 52(6):689-95.

8. Allione A, Melchio R, Martini G, Dutto L, Ricca M, Bernardi E, et al. Factors influencing desired and received analgesia in emergency department. Intern Emerg Med. 2011;6(1):69-78.

9. Farrar JT, Portenoy RK, Berlin JA, Kinman JL, Strom BL. Defining the clinically important difference in pain outcome measures. Pain. 2000;88(3):287-94.

10. Farrar JT, Young JP, LaMoreaux L, Werth JL, Poole RM. Clinical importance of changes in chronic pain intensity measured on an 11-point numerical pain rating scale. Pain. 2001;94(2):149-58.

11. World Health Organization. Cancer pain relief with a guide to opioid availability. Second ed; 1996

12. Phillips S, Gift M, Gelot S, Duong M, Tapp H. Assessing the relationship between the level of pain control and patient satisfaction. J Pain Res. 2013; 6:683-9.

13. Stahmer SA, Shofer FS, Marino A, Shepherd S, Abbuhl S. Do quantitative changes in pain intensity correlate with pain relief and satisfaction? Acad Emerg Med Off J Soc Acad Emerg Med. 1998;5(9):851-7.

14. Müller-Staub M, Meer R, Briner G, Probst M-T, Needham I. Measuring patient satisfaction in an emergency unit of a Swiss university hospital: occurrence of anxiety, insecurity, worry, pain, dyspnoea, nausea, thirst and hunger, and their correlation with patient satisfaction (part 2). Pflege. 2008;21(3):180-8

15. McNeill JA, Sherwood GD, Starck PL, Nieto B. Pain management outcomes for hospitalized Hispanic patients. Pain Management Nursing. 2001;2(1):25-36

16. Svensson I, Sjöström B, Haljamäe H. Influence of expectations and actual pain experiences on satisfaction with postoperative pain management. Eur J Pain. 2001;5(2):125-33.

17. Nagurney JT, Brown DFM, Sane S, Weiner JB, Wang AC, Chang Y. The accuracy and completeness of data collected by prospective and retrospective methods. Acad Emerg Med Off J Soc Acad Emerg Med. 2005; 12(9):884-95.

18. Rupp T, Delaney KA. Inadequate analgesia in emergency medicine. Ann Emerg Med. 2004;43(4):494-503.

19. VMS. Vroege herkenning en behandeling van pijn - VMSzorg. 2009

\section{Ready to submit your research? Choose BMC and benefit from}

- fast, convenient online submission

- thorough peer review by experienced researchers in your field

- rapid publication on acceptance

- support for research data, including large and complex data types

- gold Open Access which fosters wider collaboration and increased citations

- maximum visibility for your research: over $100 \mathrm{M}$ website views per year

At $\mathrm{BMC}$, research is always in progress.

Learn more biomedcentral.com/submissions 